\title{
Surface Segregation in AgAuCuPdPt High Entropy Alloy: Insights From Molecular Simulations
}

\author{
Chinmay Dahale, Sriram Goverapet Srinivasan*, Shashank Mishra, Soumyadipta Maiti and Beena Rai \\ TCS Research, Tata Research Development and Design Center, 54-B Hadapsar Industrial Estate, \\ Hadapsar, Pune - 411013, Maharashtra, India \\ * Corresponding author e-mail: s.goverapet@tcs.com
}

\begin{abstract}
High entropy alloys (HEAs) are emerging as a novel class of superior catalysts for diverse chemical conversions. The activity of a catalyst is intimately related to the composition and atomic structure at its surface. In this work, we used embedded atom (EAM) potential based Monte Carlo Molecular Dynamics simulations to study surface segregation in the equimolar AuAgCuPdPt HEA, that was recently shown to be an efficient catalyst for $\mathrm{CO}_{2}$ electrochemical reduction. Firstly, EAM potentials were extensively validated against experimental segregation data for several different binary and ternary compositions. Subsequently, simulations on the HEA were carried out for four different surface orientations, spherical and cubical nanoparticles, to obtain detailed structural and concentration profiles normal to the surface. In all cases, Ag atoms were found to preferentially segregate to the surface while the subsurface layer mainly consisted of Au atoms. No Pt atoms were found on the surface layer for all systems. A detailed analysis neighborhood of each surface site revealed that the atoms formed a finite number of chemically unique clusters. The percentage of chemically unique sites were larger for elements with lower concentration at the surface. Together with the physical diversity surrounding each site, the enrichment of one or more element(s) at the surface also increased its number of unique catalytically active sites. Results from our work suggest that HEAs are prone to surface segregation and such effects must be taken into consideration while modeling the surface chemistry of these materials.
\end{abstract}

\section{Introduction}

High entropy alloys (HEAs) containing multiple principal components in a nearly equi-atomic ratio [1, 2], are emerging as a superior class of structural and functional materials [3]. HEAs have depicted superior mechanical properties such as simultaneously possessing high strength and ductility over a wide temperature range $[4,5]$, good wear resistance [6], higher fracture toughness at cryogenic conditions [7] etc. The origin of such superior mechanical properties seem to be closely related to the microstructure at the atomic scale, such as short-range ordering / clustering (SRO/SRC) wherein atoms rearrange to form periodic nanodomains that are 'rich' in one or two elements [8,9]. In addition to displaying excellent mechanical properties, HEAs have shown functional properties such as improved tolerance to irradiation $[10,11]$ and hydrogen embrittlement [12], better corrosion resistance [13, 14], superconductivity [15] etc.

More recently, these materials are being explored as heterogeneous catalysts for diverse chemical conversions [16, 17]. Unlike conventional mono-metallic catalysts, where each binding site is surrounded by the same kind of atoms, the presence of multiple elements in HEAs provides a wide variety of binding sites $[18,19]$. Each site contains a unique combination of neighboring elements that causes changes to the local electronic structure of the active site. As a result, HEA surfaces could present a distribution of adsorbate binding energies as opposed to the single value obtained at the surface of mono-metallic catalysts. Such a distribution of adsorbate binding energies provides a unique opportunity to tune the composition of these alloys to obtain desired binding energy distribution to steer catalytic selectivity 
towards one or more products $[18,19]$. However, like nanoclustering in HEA bulk causing SRC, preferential surface segregation of one or more elements could limit the number of 'unique' sites that may be observed on the surface of these HEAs. In fact, earlier electrochemical studies on the corrosion resistance of HEAs have shown the evolution of multiphase structure with significant elemental segregation [13]. Thus, an understanding of the surface segregation in such alloys becomes essential, particularly from the standpoint of 'surface-governed' applications such as catalysis. Inclusion of surface segregation as an intermediate step in the optimization of HEA composition for any chemical conversion, could then yield a more realistic screening of the vast compositional space of HEAs.

A few studies in the past have addressed segregation phenomena in HEAs, both at surfaces and at grain boundaries [20-25]. Much of these studies have been carried out on the cantor alloy (FeMnNiCoCr FCC HEA). These studies revealed that segregation at the grain boundaries was strongly dependent on the nature of these boundaries [21]. While no segregation or very little Ni enrichment was observed at low energy grain boundaries, significant $\mathrm{Ni}$ enrichment with $\mathrm{Mn}$ co-segregation was observed at general high angle grain boundaries [21]. Mn was the dominant element on the (001), (110) and (320) surfaces of the HEA [22, 25]. A lower surface energy of Mn and a strongly negative mixing enthalpy of the Ni-Mn pair was attributed to such elemental segregation $[22,25]$. While the quantitative (and to some extent qualitative) amounts of the segregating species may differ across these reports, all of them point to HEAs being prone to segregation and that such effects must be taken into account for a more thorough and realistic understanding of surface governed phenomena on HEAs.

In this work, we have used EAM based Monte Carlo/Molecular Dynamics (MC/MD) simulations to study surface segregation in AuAgCuPdPt FCC HEA that was recently shown to be an active catalyst for $\mathrm{CO}_{2}$ reduction $[26,27]$. Prior to simulations on the HEA, the EAM potential was extensively validated against experimental segregation data reported for binary and ternary alloys formed from elements in our HEA. Subsequently, MC/MD simulations were carried out on 4 different surface orientations and two different nanoparticle shapes of the HEA. The results from these simulations were used to obtain detailed compositional depth profiles as well as the number and kinds of different active sites on each of these surfaces. Implications of these findings for surface catalysis on these HEAs are discussed.

\section{Computational Methods and Details}

MC/MD simulations were used to investigate surface segregation in AgAuCuPdPt FCC HEA. The pairwise interaction between atoms and embedding functions of the elements was described using the EAM potential of Zhou et al [28]. This potential was firstly validated against experimental surface segregation data on a few binary and ternary alloys formed from the components in our HEA. The lattice parameter for each of these alloys was taken as a simple average of the lattice parameters of the constituent elements. Cubic systems containing about 6000 atoms were built using these lattice parameters for each of the alloys following which the systems were energy minimized while allowing for the lattice parameters and the positions of the atoms to relax. Subsequently, NPT simulations were carried out at the target temperature and 1 bar pressure using a molecular dynamics (MD) timestep of $1 \mathrm{fs}$. MD simulations were run for a duration of $50 \mathrm{ps}$. The value of the lattice parameter at the target temperature was then obtained as an average over the last $15 \mathrm{ps}$ of simulation. This lattice parameter was used to construct 2D periodic slabs of suitable orientation for each of these alloys. The (111), (100) and (110) surfaces contained 14, 16 and 18 atomic layers respectively. MC/MD simulations following earlier established protocol was used to evolve these systems [29]. Briefly, two different atoms were randomly chosen and their positions were swapped. The atomic coordinates were then relaxed using the conjugate gradient scheme. The 'acceptance' of the new atomic coordinates was decided based on the Metropolis criterion; the atomic swap was immediately accepted if it caused a reduction in the potential energy of the 
system, else it was accepted with a probability of $e^{\frac{-\Delta U}{k_{B} T}}$, where $\Delta \mathrm{U}$ was the difference in the potential energies, $k_{B}$ was the Boltzmann constant and $T$ was the temperature. During the MC/MD simulations, atoms in the top 6 layers alone were allowed to be swapped while the atoms in the layers beneath them were held frozen to mimic the bulk material. The surface evolution was carried out for a total of 10 swaps per atom. Three different configurations were considered for each system. At the end of the MC/MD simulations, the composition at the outermost layer (surface layer) was obtained as an average over the three configurations. The surface compositions of various alloys obtained from our simulations closely matched the experimental data, as discussed in the results section.

Following validation of the EAM potential, MC/MD simulations were carried out to study surface segregation in the HEA. Two different nanoparticle (NP) shapes, cubic and spherical, and four different surface orientations, namely (100), (110), (111) and (211), were studied. All atoms were allowed to be swapped for the two nanoparticles while atoms in the top 6 atomic layers of the (100), (110) and (111) surfaces, and those in the top 12 layers of the (112) surface alone were allowed to be swapped. Five configurations with different random arrangement of atoms were considered for each system. All the results were averaged over the five different initial configurations. MC/MD system evolution was performed at a temperature of $1200 \mathrm{~K}$ till 10 swaps per atom for the surfaces and 15 swaps per atom for the NPs. All the molecular simulations in this work were performed using the LAMMPS software [30] while the images of the atomic structures were generated using the VMD package [31].

Depth-wise and radial compositional profiles were computed for the surface and NPs, respectively. While these profiles give us the average alloy composition in successive layers, knowledge of the distribution of various kinds of active sites is essential from a catalyst perspective. Accordingly, the systems were analyzed to obtain various chemically unique sites and their distribution at the surface. Each surface atom was encoded based on the identities of its nearest neighbor elements. Then, the set of all unique encodings gave the total number of unique active sites at the surface while the average number of occurrences of each of these encodings gave the distribution of these sites.

\section{Results}

\section{Segregation in Binary and Ternary Alloys}

Segregation of elements in binary and ternary alloys obtained from our EAM potential was compared against experimentally reported surface compositions to validate both the EAM potential and the MC/MD protocol. Table 1 summarizes the results from these simulations. The simulation results are an average over three random configurations for each system while the error corresponds to one standard deviation about the mean. Clearly, the composition at the surface obtained from our MC/MD simulations closely matches those from the experiments at diverse conditions and alloy compositions.

Table 1: Comparison of experimental and theoretical surface compositions post segregation in various binary and ternary alloys. The error bars correspond to one standard deviation about the mean value.

\begin{tabular}{|c|c|c|c|c|c|c|}
\hline System & $\mathbf{T}(\mathbf{K})$ & Surface Plane & $\begin{array}{c}\text { Segregating } \\
\text { phase }\end{array}$ & $\begin{array}{c}\text { Expt. Value } \\
(\boldsymbol{\%})\end{array}$ & $\begin{array}{c}\text { MC/MD Value } \\
(\%)\end{array}$ & Ref. \\
\hline $\mathrm{Ag}_{0.33} \mathrm{Pd}_{0.67}$ & 820 & $(111)$ & $\mathrm{Ag}$ & 94.8 & $98.26 \pm 0.56$ & {$[32]$} \\
\hline $\mathrm{Ag}_{0.33} \mathrm{Pd}_{0.67}$ & 700 & $(100)$ & $\mathrm{Ag}$ & 98.6 & $99.5 \pm 0.41$ & {$[32]$} \\
\hline
\end{tabular}




\begin{tabular}{|c|c|c|c|c|c|c|}
\hline $\mathrm{Pd}_{0.75} \mathrm{Pt}_{0.25}$ & 600 & $(111)$ & $\mathrm{Pd}$ & 99.0 & $99.91 \pm 0.12$ & {$[33]$} \\
\hline $\mathrm{Pd}_{0.75} \mathrm{Cu}_{0.25}$ & 600 & $(111)$ & $\mathrm{Cu}$ & 26.0 & $42.0 \pm 0.24$ & {$[33]$} \\
\hline $\mathrm{Ag}_{0.3} \mathrm{Au}_{0.7}$ & 973 & $(111)$ & $\mathrm{Ag}$ & 40.0 & $33.25 \pm 2.24$ & {$[34]$} \\
\hline $\mathrm{Au}_{0.59} \mathrm{Cu}_{0.41}$ & 723 & $(111)$ & $\mathrm{Au}$ & 98.0 & $82.0 \pm 0.58$ & {$[34]$} \\
\hline $\mathrm{Au}_{0.71} \mathrm{Cu}_{0.29}$ & 698 & $(111)$ & $\mathrm{Au}$ & 98.0 & $94.75 \pm 0.93$ & {$[34]$} \\
\hline $\mathrm{Au}_{0.9} \mathrm{Pt}_{0.1}$ & 873 & $(111)$ & $\mathrm{Au}$ & 100.0 & $99.84 \pm 0.13$ & {$[34]$} \\
\hline $\mathrm{Ag}_{0.091} \mathrm{Cu}_{0.303} \mathrm{Pd}_{0.606}$ & 1000 & $(110)$ & $\mathrm{Ag}$ & $28.0 \mathrm{Cu}$ & $25.4 \pm 0.34 \mathrm{Cu}$ & {$[35]$} \\
\hline
\end{tabular}

The evolved surface structures of these alloys are shown in figure S1 of the supporting information. On the (111) surface of $\mathrm{Ag}_{0.33} \mathrm{Pd}_{0.67}$ alloy, the minority Pd were mostly present as isolated sites while a few of them were present as nearest neighbor double sites or next-nearest neighbor double sites. (see fig S1a and S1b) Such distribution of Pd atoms at the surface is in excellent agreement with prior STM measurements [32]. Thus, the EAM potential and the MC/MD protocol can reproduce not just the compositional, but also structural features at the surface. In other alloys that were significantly enriched in one component at the surface (such as $\mathrm{Pd}_{0.75} \mathrm{Pt}_{0.25}, \mathrm{Au}_{0.71} \mathrm{Cu}_{0.29}$ and $\mathrm{Au}_{0.9} \mathrm{Pt}_{0.1}$ ), the minority element was mainly present as isolated sites (see figs $\mathrm{S} 1 \mathrm{~d}, \mathrm{~S} 1 \mathrm{~h}$ and $\mathrm{S} 1 \mathrm{i}$, respectively).

\section{Segregation in Spherical and Cubical HEA NPs}

Having validated the EAM potential and the MC/MD simulation protocol, we simulated surface segregation in the AgAuCuPdPt FCC HEA. We first studied segregation in a spherical NP of radius 2.64 $\mathrm{nm}$ containing 5775 atoms. All the atoms in the NP were randomly selected for attempted MC swaps, without any demarcation of surface and bulk layers. Unlike planar surfaces that are periodic along the two basal plane directions, NPs are non-periodic, isolated entities. As a result, each atom at the surface may not only witnesses a different chemical environment imposed by its neighbors but also a different number of neighbors (i.e., coordination number $(\mathrm{CN})$ ) itself. Figure 1 shows images of the spherical NP formed by random distribution of atoms (i.e., prior to MC/MD simulations) as well as the evolved structure while the compositional variation in the NP as a function of radius is presented in Fig. 2. 

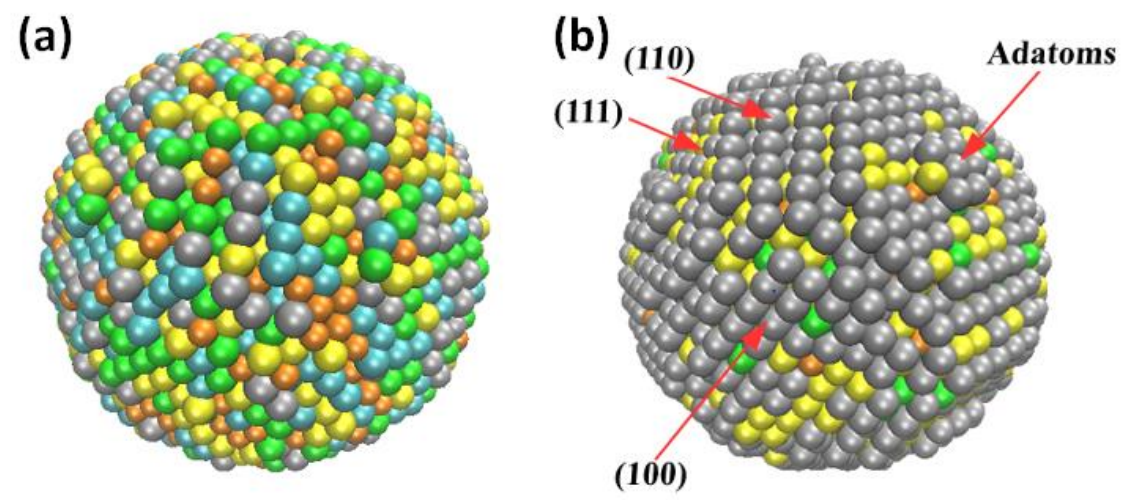

Figure 1: Structure of a spherical HEA NP. (a) Initial structure where atoms are randomly distributed and (b) Segregated structure after the MC/MD simulations. Gray, yellow, brown. Green and blue color spheres represent $\mathrm{Ag}, \mathrm{Au}, \mathrm{Cu}, \mathrm{Pd}$ and $\mathrm{Pt}$ atoms respectively.
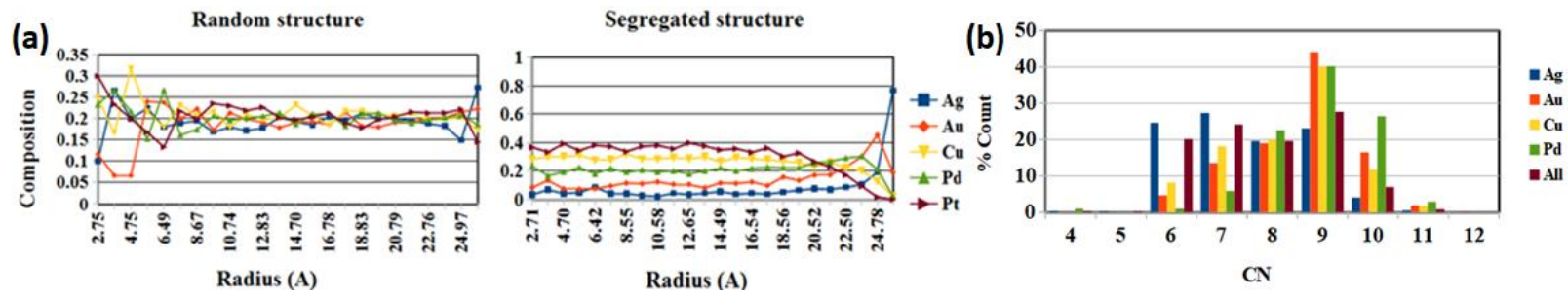

Figure 2: (a) Variation in the composition of the spherical NP as a function of the radius. (b) Distribution of the coordination numbers of various atoms at the surface of the segregated NP.

Clearly a significant amount of atom migration and segregation can be observed in the NP. The surface of the NP predominantly consists of Ag atoms, whose concentration drops drastically as we start moving towards the inner regions of the NP. Au atoms are the $2^{\text {nd }}$ most abundant at the surface while trace amounts of $\mathrm{Cu}$ and $\mathrm{Pd}$ atoms are also seen. No Pt atoms were present at the surface of the NP. Au atoms were the most abundant in the $1^{\text {st }}$ subsurface layer with $\mathrm{Ag}$ and $\mathrm{Pd}$ atoms present in nearly equal amounts. Interestingly, all the compositional variations seem to occur within a $1 \mathrm{~nm}$ region beneath the surface of the NP. Beyond this region, the composition remains almost a constant till the center of the NP. Thus, the 'core' of the NP is a random alloy of a fixed composition with concentration gradations occurring only in the near surface region. Examination of the atomic structure reveals that the surface of the evolved NP consists of (111), (100) and (111) terraces. The Au atoms at the surface, that are the $2^{\text {nd }}$ most abundant in proportion, seem to mainly form contiguous islands as opposed to being distributed as isolated atoms. Furthermore, they seem to be present mainly on the (100) and (111) facets. The CN distribution (Fig 2b) shows that overall, the surface atoms have a CN varying between 6 and 10. While majority of the $\mathrm{Au}, \mathrm{Cu}$ and $\mathrm{Pd}$ atoms have a $\mathrm{CN}$ of 9, a larger fraction of $\mathrm{Ag}$ atoms have a lower coordination number of 6 and 7. Thus, not only do we see a preferential enrichment of Ag at the surface, but these $\mathrm{Ag}$ atoms also present a wide range of $\mathrm{CNs}$, indicating that a diverse set of $\mathrm{Ag}$ sites are present at the surface.

To further explore the HEA NP shape effects, we simulated segregation in a cubical NP with an initial edge length of $42.57 \AA$, containing 6083 atoms. Unlike a spherical NP, each of the 6 faces of a cubical NP belong to the same family of surface orientations (i.e., $\{100\}$ family of planes). Thus, other than the atoms at the edges and corners of the NP, rest of the atoms have the same number of nearest 
neighbors. Figure 3 shows the structure of the cubical NP before and after the MC/MD evolution while figure 4 shows the average composition at the surface of the NP.
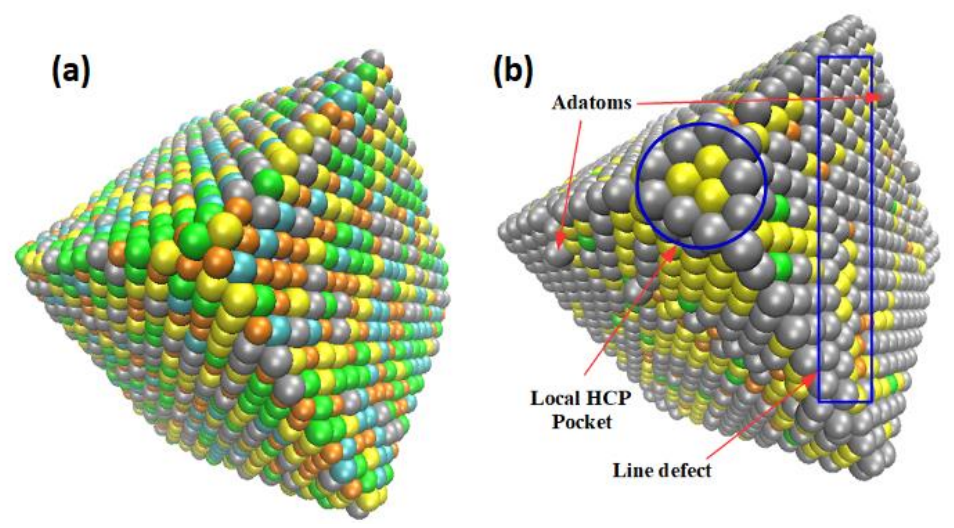

Figure 3: Structure of a cubical HEA NP. (a) Initial structure where atoms are randomly distributed and (b) Segregated structure after the MC/MD simulations. Gray, Yellow, Brown. Green and Blue color spheres represent $\mathrm{Ag}, \mathrm{Au}, \mathrm{Cu}, \mathrm{Pd}$ and $\mathrm{Pt}$ atoms respectively.

\section{Cubical NP Composition}

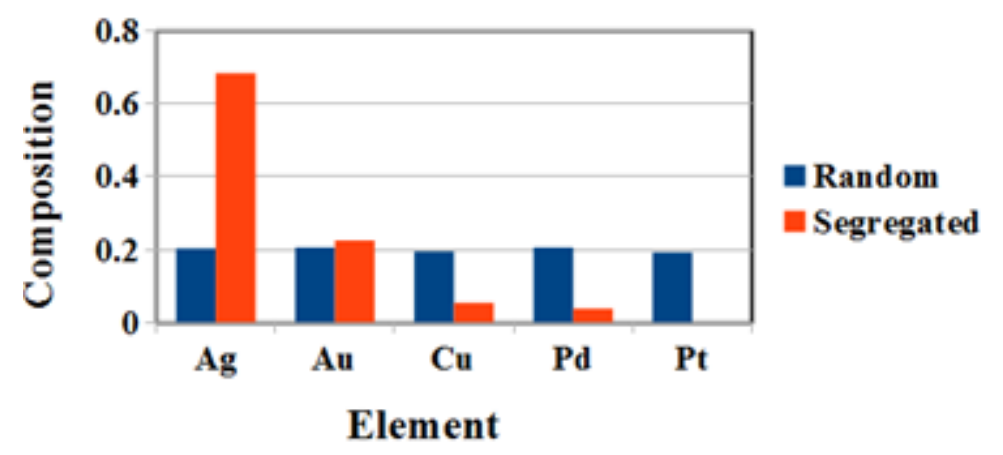

Figure 4: Average surface composition of a cubical HEA NP.

Once again, we notice that the average composition at the cubical NP surface is very similar to that of the spherical NP with Ag being the dominant element followed by $\mathrm{Au}$. $\mathrm{Cu}$ and $\mathrm{Pd}$ were present in smaller quantities and no Pt atoms were seen at the surface. Au atoms at the surface clustered together to form contiguous islands that are far more prominent on the cubical NP. A marked difference in the structural features between the random and segregated HEA is also observed. The corners of the NP are smoothened in the segregated structure to form a local (111) like terrace that bridges the adjacent three (100) like terraces. The preferential migration of elements with smaller atomic radii (such as $\mathrm{Pt}$ and $\mathrm{Cu}$ ) to the interiors caused the NP to shrink, with the edge length reducing to $40.96 \AA$. The resultant atomic strain on the dominant $\mathrm{Ag}$ atoms (having the largest atomic radii among the 5 elements) at the surface caused a few of these atoms to leave their lattice sites and form popped-up 'adatoms' on the surface. Consequently, line defects (shown by blue rectangle in Fig 3b) were seen on the (100) terrace, as atoms relaxed from their lattice sites to partially fill the void caused by the departure of the adatom. Such reconstruction increased the roughness of the (100) terraces. While the 'cleaved' NP with random distribution of atoms had atomically flat terraces, upon segregation, the average roughness of the (100) terraces (computed as one standard deviation about the mean value) increased to $0.59 \AA$. Thus, 
segregation in the HEA NPs resulted in surfaces with structurally richer features albeit with lower chemical diversity.

\section{Segregation in HEA surfaces}

Following simulations on NPs, we studied segregation in extended surfaces of the HEA. Specifically, MC/MD simulations were carried out on the (100), (110), (111) and (211) surfaces. Simulations on the NPs suggested that the variation in the composition occurs mainly over a $1 \mathrm{~nm}$ region beneath the surface. Thus, for the MC/MD simulations on the surfaces, atoms in the top 6 layers alone were swapped. Although no swaps were allowed among the remaining atoms, during geometry optimization in the MC/MD protocol, the positions of all the atoms in the system were relaxed. In each case, the system was evolved upto $10 \mathrm{MC}$ swaps per atom. The evolution of the surface composition as a function of the number of swaps is shown in Figure $\mathrm{S} 2$ of the supporting information for all the four surfaces. Table 2 presents the composition at the surface for all four orientations while Figure 5 shows the depth-wise composition profiles for the initial random and evolved structures.

Table 2: Surface composition of the evolved HEA along four different orientations. The error bars correspond to one standard deviation about the mean value.

\begin{tabular}{|c|c|c|c|c|c|}
\hline Plane & $\mathbf{A g}(\%)$ & $\mathbf{A u}(\%)$ & $\mathbf{C u}(\%)$ & Pd (\%) & Pt (\%) \\
\hline$(100)$ & $73.46 \pm 2.11$ & $20.40 \pm 1.40$ & $4.33 \pm 0.96$ & $1.78 \pm 0.83$ & $0.0 \pm 0.0$ \\
\hline$(110)$ & $78.40 \pm 2.22$ & $12.45 \pm 1.36$ & $6.50 \pm 1.27$ & $2.60 \pm 0.60$ & $0.0 \pm 0.0$ \\
\hline$(111)$ & $62.88 \pm 1.70$ & $22.06 \pm 0.80$ & $9.95 \pm 1.32$ & $5.01 \pm 0.94$ & $0.0 \pm 0.10$ \\
\hline$(211)-\mathrm{L} 1$ & $84.56 \pm 1.35$ & $11.11 \pm 0.70$ & $3.70 \pm 0.70$ & $0.61 \pm 0.93$ & $0.0 \pm 0.0$ \\
\hline$(211)-\mathrm{L} 2$ & $56.42 \pm 1.98$ & $31.11 \pm 1.54$ & $6.91 \pm 3.06$ & $5.56 \pm 1.79$ & $0.0 \pm 0.0$ \\
\hline$(211)-\mathrm{L} 3$ & $31.36 \pm 1.18$ & $37.04 \pm 1.15$ & $16.42 \pm 3.82$ & $14.20 \pm 2.99$ & $0.99 \pm 0.33$ \\
\hline
\end{tabular}

Among the four surfaces, the (100), (110) and (111) surfaces are atomically flat with each surface atom coordinated to 8, 7 and 9 nearest neighbors. (211) on the other hand, is a stepped surface that can be considered as having a step edge (L1), upper edge (L2) and a lower edge (L3) with 7, 9 and 10 coordination numbers, respectively. On all four surfaces, akin to NPs, Ag was the predominant element at the surface. Its concentration dropped rapidly in the subsurface region and reached a constant value as we moved deeper into the material. Au was the $2^{\text {nd }}$ most prevalent element on the surface layer for all four orientations. Its concentration reached a peak in the sub-surface layer for the (100), (110) and (111) orientations while this peak occurred in the L3 layer for the (211) surface. Pt was absent at the surface for all four orientations. However, its concentration increased gradually to become the dominant element deeper with the material. Interestingly, the concentration of Pt in the sub-surface layer was more pronounced on the (111) surface with a higher packing density compared to (110) or (211) surfaces with a lower packing density. $\mathrm{Cu}$ and $\mathrm{Pd}$ were seen in small quantities at the surface, with their concentration increasing gradually to reach a constant value in deeper regions. While Ag was the predominant element at the surface, its percentage composition was higher on surfaces with lower packing density (e.g., (211) or (110), see table 2). Atomic structures of the segregated systems are shown in Figure S3 of the supporting information. Unlike cubical NP, no defects were formed on any of the four surfaces upon segregation. 

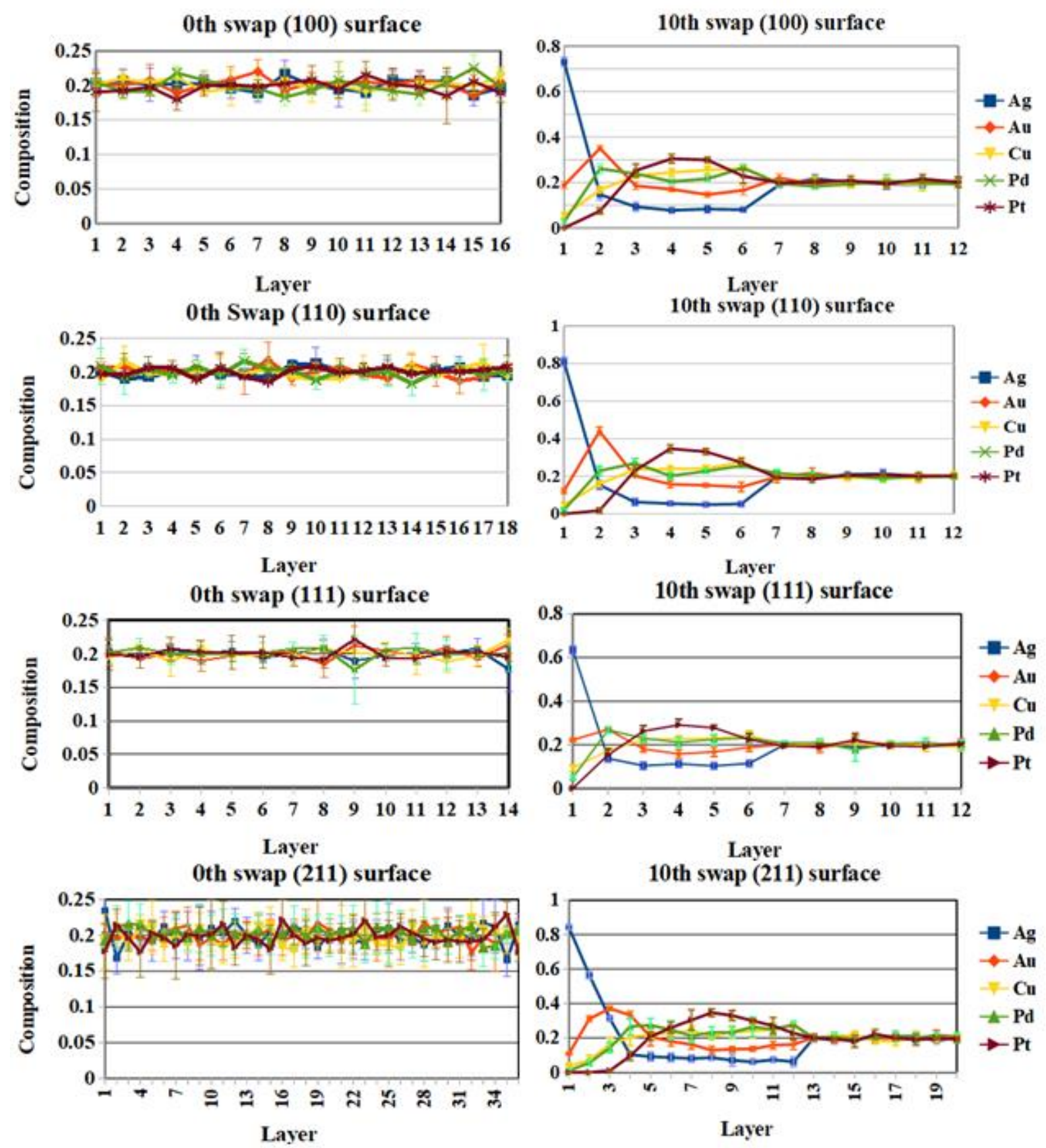

Figure 5: Depth wise compositional profiles for (100), (110), (111) and (211) surfaces. The left column figures correspond to the initial structure with random distribution of atoms while the right column figures correspond to the evolved structures. The error bars correspond to one standard deviation about the mean value.

Overall, in both NPs and extended surfaces, $\mathrm{Ag}$ and $\mathrm{Au}$ elements preferentially migrated to the surface, $\mathrm{Pt}$ atoms remained distributed in the bulk region while $\mathrm{Cu}$ and $\mathrm{Pd}$ were present in both bulk and near surface regions. Such spatial preferences for these atoms closely follow the order of the surface energies of the pure metals. It is now well known that for all the three surfaces, (100), (110) and (111), the surface energies follow the order: $\mathrm{Ag}<\mathrm{Au}<\mathrm{Cu}<\mathrm{Pd}<\mathrm{Pt}$ [36]. Accordingly, elements with lowest 
surface energy ( $\mathrm{Ag}$ and $\mathrm{Au}$ ) preferred to segregate near surface while that with the highest surface energy (Pt) preferred to remain in the bulk.

\section{Nature of Surface Sites}

Compositional analysis reported in the previous section provides information on the kinds and quantities of various atoms at the surface. However, rationalizing experimentally observed catalytic efficiencies as well as designing newer catalysts require detailed knowledge of the specific arrangement of atoms at the surface. Thus, we have carried out neighborhood analysis on the evolved structures to identify chemically unique environments around each surface site and quantified the percentage of their occurrence. On the (100) and (111) surfaces, the neighborhood of each atom at the surface was encoded using a 10 digit number. Every site on the (100) surface contains 4 neighbors each on the surface and subsurface layer for a total of 8 nearest neighbors. On the (111) surface, each site has 6 and 3 neighbors in the surface and sub-surface layer respectively, for a total of 9 nearest neighbors. The first 5 digits of the encoding represented the number of atoms of $\mathrm{Ag}, \mathrm{Au}, \mathrm{Cu}, \mathrm{Pd}$ and $\mathrm{Pt}$ kinds (in that order) among the surface neighbors of a given site while the last five digits represented their numbers among the subsurface neighbors. For example, an encoding such as 4000003100 for a site would imply that the site is surrounded by $4 \mathrm{Ag}$ atoms in the surface layer and $3 \mathrm{Au}, 1 \mathrm{Cu}$ atom in the subsurface layer, i.e., (4Ag)$(3 \mathrm{Au}, 1 \mathrm{Cu})$ neighborhood. Sites on the (110) surface were encoded by a 15 digit number; the first 5 digits representing the two nearest neighbors on the surface, the next 5 digits representing the 4 neighbors in the sub-surface layer and the last 5 digits corresponding to the neighbor in the $2^{\text {nd }}$ subsurface layer. Finally, sites on the L1, L2 and L3 edges of the (211) surface as well as those on the NPs were encoded using a 5digit number that accounted for various kinds of atoms among its neighbors. A surface site was considered 'unique' if its encoding was different from the encodings of all other sites.

Table 3 presents the results from the neighborhood analysis on both the extended surfaces as well as NPs. Note that the table does not contain any details for Pt since these atoms were not present on the surface in any of the six systems. The numbers report the cumulative counts from all the 5 configurations studied for each system.

Table 3: Statistics on the number of sites of different kinds on the surfaces of various systems.

\begin{tabular}{|c|c|c|c|c|c|c|c|c|}
\hline SiteslSurface & $(100)$ & $(110)$ & $(111)$ & $(211)-L 1$ & $(211)-L 2$ & $(211)-L 3$ & Spherical NP & Cubical NP \\
\hline \multicolumn{8}{|c|}{ Ag site at the surface } \\
\hline Total sites & 1431 & 1367 & 1370 & 682 & 457 & 254 & 3872 & 4813 \\
\hline Unique sites & 366 & 410 & 447 & 103 & 154 & 121 & 529 & 589 \\
\hline \% Unique sites & 25.57 & 29.99 & 32.62 & 15.1 & 33.69 & 47.63 & 13.66 & 12.23 \\
\hline \multicolumn{8}{|c|}{ Au site at the surface } \\
\hline Total sites & 368 & 207 & 480 & 88 & 252 & 300 & 914 & 1707 \\
\hline Unique sites & 211 & 147 & 307 & 54 & 132 & 166 & 365 & 475 \\
\hline \% Unique sites & 57.33 & 71.01 & 63.95 & 61.36 & 52.38 & 55.33 & 39.93 & 27.82 \\
\hline \multicolumn{8}{|c|}{ Cu site at the surface } \\
\hline Total sites & 108 & 80 & 202 & 32 & 56 & 133 & 110 & 404 \\
\hline Unique sites & 88 & 64 & 162 & 25 & 43 & 104 & 91 & 188 \\
\hline \% Unique sites & 81.48 & 80 & 80.19 & 78.12 & 76.78 & 78.19 & 82.72 & 46.53 \\
\hline \multicolumn{8}{|c|}{ Pd site at the surface } \\
\hline Total sites & 53 & 36 & 107 & 8 & 45 & 115 & 102 & 283 \\
\hline Unique sites & 45 & 32 & 97 & 8 & 40 & 89 & 85 & 155 \\
\hline \% Unique sites & 84.90 & 88.88 & 90.65 & 100 & 88.88 & 77.39 & 83.33 & 54.77 \\
\hline
\end{tabular}


Table 3 provides interesting insights into the segregation pattern of elements on the surface. Firstly, we notice that while $\mathrm{Ag}$ is the most abundant element at any surface, most of the Ag atoms reside in a chemically similar neighborhood. Secondly, as the concentration of an element reduces at the surface, the percentage of its unique sites increases. However, the extent of this increase seems to be lower on cubical NPs as compared to extended surfaces or spherical NPs. While about $80 \%$ of the Pd and Cu sites were chemically unique on these surfaces, only $\sim 50 \%$ of these sites were unique on the cubical NP, pointing to the fact that like atoms were more clustered on the cubical NP as compared to extended surfaces or spherical NP (see Figs 2 and 3 for NPs and Fig S3 for extended surfaces).

The percentage occurrence of various kinds of chemical neighborhoods for each elemental site on the (111) surface is shown in Figure 6 while this distribution for the remaining systems is presented in Figures S4-S10 of the supporting information. Tables S1 to S33 in the supporting information enlists the encoding corresponding to each index in the X axis of Figure 6 and Figures S4-S10.

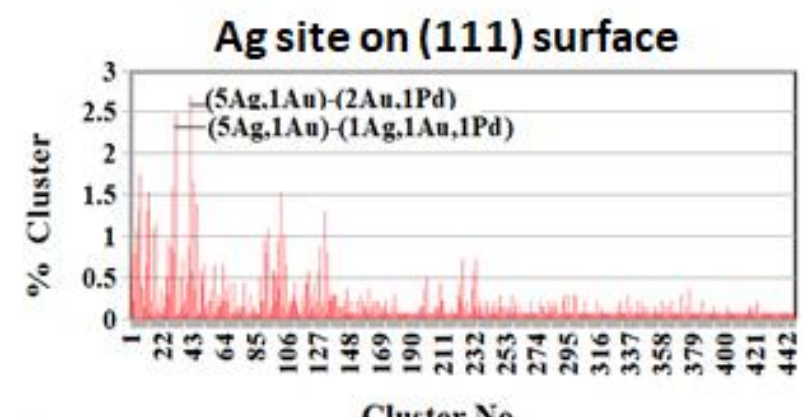

Cluster No.

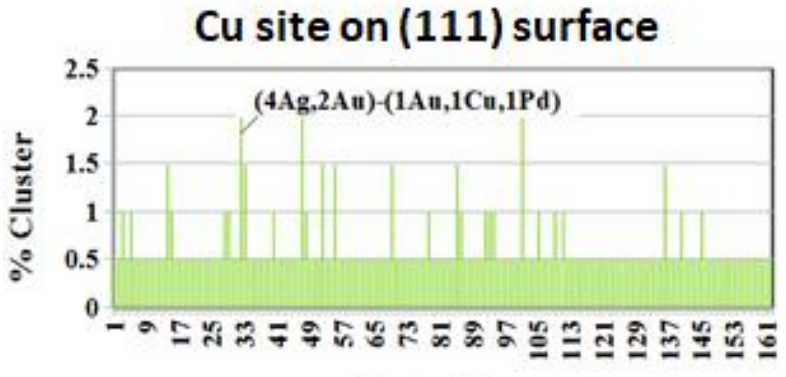

Cluster No.

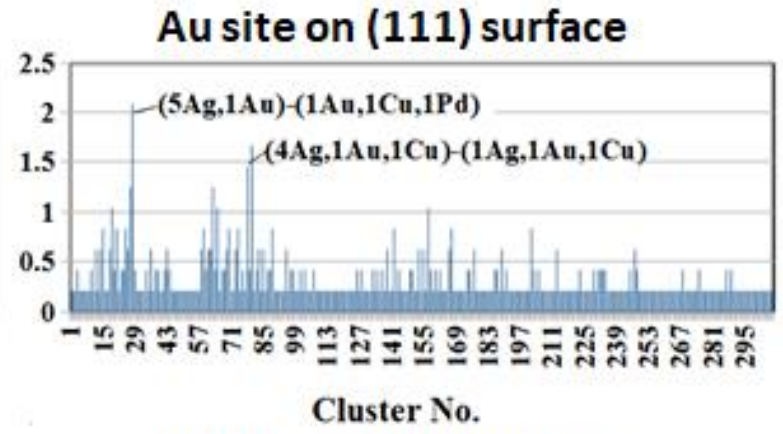

Pd site on (111) surface

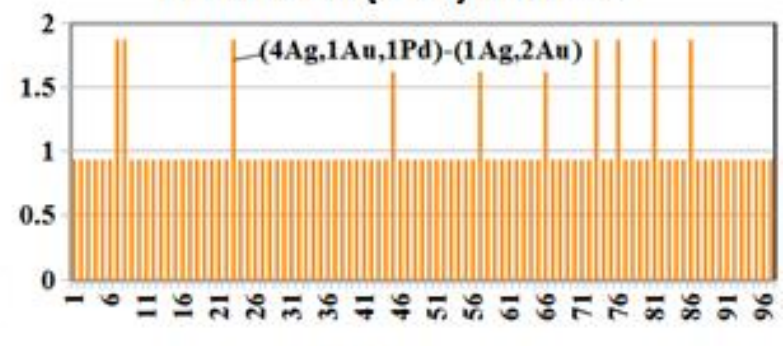

Cluster No.

Figure 6: Percentage distribution of various unique chemical neighborhoods for each elemental site on the (111) surface.

From Figure 6, it is clear that the dominant neighborhood of each elemental site contains a majority of $\mathrm{Ag}$ atoms, consistent with the fact the segregated surface is rich in Ag. A similar scenario is observed for other surface terminations as well as the two NPs, as shown in Figures S4-S10.

\section{Discussion}

Molecular simulations were used to study the surface segregation in equimolar AuAgCuPdPt FCC HEA. Simulations were carried out using an EAM potential on both NPs as well as extended surfaces. Prior to these simulations the EAM potential was extensively validated against experimental surface segregation data on binary alloys and a ternary alloy formed from the elements constituting the HEA. In all these cases, the potential was able to closely reproduce the experimentally measured surface 
compositions. In addition, on the $\mathrm{Pd}_{0.67} \mathrm{Ag}_{0.33}$ (111) surface, the simulated distribution of atomic configurations was in excellent agreement with the STM data [32]. MC/MD simulations on the HEA revealed that both NPs and extended surfaces of this alloy are prone to segregation. In all cases, Ag atoms were significantly enriched at the surface while the sub-surface layer was rich in Au atoms. The concentration of Ag dropped rapidly in the sub-surface layers. In contrast, no Pt atoms were observed at the surface of any of the systems. Their concentration gradually increased from the subsurface layers to reach a maximum value in the bulk. Similarly, only trace amounts of $\mathrm{Cu}$ and $\mathrm{Pd}$ atoms were seen on the surface. The gradation in the elemental composition was seen only within a $\sim 1 \mathrm{~nm}$ region beneath the surface, beyond which it remained a constant. Thus, segregation in this alloy, as exemplified by the spherical NP, resulted in two regions: (a) a near surface region with significant variations in the elemental composition and (b) a bulk region of a fixed alloy composition that was enriched (i.e., >20\%) in Pt and $\mathrm{Cu}$, and depleted in $\mathrm{Ag}$ and $\mathrm{Au}$ (i.e., < 20\%).

The driving force (or the absence of it), $\Delta H_{\text {seg }}$, for surface segregation is mainly thought to arise from a competition between three factors: (i) the surface energy of the individual elements, (ii) interactions between elemental pairs which is proportional to the mixing enthalpy and (iii) the elemental strain energy due to a mismatch in the lattice dimensions between the alloy and the pure elements [37]. In addition to these, site-competition effects could also affect the segregation tendencies in a multicomponent alloy [38]. Thus, the combination of factors leading to the observed surface segregation is dependent not only on the alloy composition but also on the nature of the surface/interface. In the case of the AgAuCuPdPt FCC HEA studied in this work, Ag has the lowest (100), (110) and (111) surface energies followed by $\mathrm{Au}$ [36]. Further, the atomic strain, obtained as the difference in the computed elemental radius between the pure element and the HEA, is also largest for $\mathrm{Ag}(0.005 \mathrm{~nm})$ and $\mathrm{Au}(0.004$ $\mathrm{nm})$. A combination of these two factors causes the enrichment of $\mathrm{Ag}$ and $\mathrm{Au}$ at the surface, which is further aided by a favorable mixing enthalpy $(-5.544 \mathrm{~kJ} / \mathrm{mol})$. Although the surface energies of $\mathrm{Cu}$ and $\mathrm{Pd}$ are much higher than those of $\mathrm{Ag}$ and $\mathrm{Au}$, the presence of small amounts of these elements at the surface could be explained by a strongly negative mixing enthalpy between Ag-Pd ( $-7.361 \mathrm{~kJ} / \mathrm{mol})$ and $\mathrm{Au}-\mathrm{Cu}(-8.803 \mathrm{~kJ} / \mathrm{mol})$. Indeed, cluster analysis revealed the presence of at least one $\mathrm{Ag}(\mathrm{Au})$ in the neighborhood of almost all the surface $\mathrm{Pd}(\mathrm{Cu})$ atoms. Such segregation dependency on the surface energy of pure elements and pairwise negative mixing enthalpy is also supported by the experimental study in Cantor alloy [25]. It appears that a factor like relative diffusivity of elements in the HEA matrix has no significant role in the thermodynamics driven surface segregations in HEAs and other alloys [25, 32-35]. The computed atomic strain and the binary mixing enthalpies based on the Miedema model [3941] are presented in table S34 and S35 of the supporting information, respectively.

While compositional analysis provided information on the predominant species on the surface, the specific arrangement of various atoms at the surface could have profound implications for the catalytic activity of an HEA. Thus, we carried out cluster analysis to identify the chemical neighborhood around each site at the surface. Our analysis showed that while Ag is the predominant element at the surface, not every Ag site is chemically identical. Depending on the system, about 12 to $48 \%$ of the $\mathrm{Ag}$ atoms at the surface contained a chemically different neighborhood. This number was larger for the $2^{\text {nd }}$ most abundant element $(\mathrm{Au})$. As the concentration of an element at the surface reduced, more of these atoms had a chemically distinct neighborhood. The presence of a vast variety of unique sites is thought to underlie the enhanced efficiency of HEA catalysts. With random distribution of all constituent elements at the surface, the HEA is expected to present a binding energy distribution for an adsorbate, containing as many peaks as the number of elements in the alloy. The peak integral (or area under the curve) provides a measure of the number of active sites of that element type [19]. However, with surface segregation, the number of unique elements at the surface might be less than that in the bulk alloy. In our case, we notice 
that no Pt atoms were present at the surface for any of the 6 systems studied. On the other hand, enrichment of the surface in one of more elements could increase the number of unique sites arising from that element. This can be understood better by considering Ag atoms on the (111) surface in our simulations. The (111) surface contained 432 atoms. Assuming a random distribution of atoms, $20 \%$ of these sites would be occupied by $\mathrm{Ag}$, all of which are potentially unique (i.e., 86 unique $\mathrm{Ag}$ sites on the surface). Upon segregation, we noticed that $\sim 63 \%$ of the sites were occupied by Ag of which $\sim 33 \%$ were chemically unique (i.e., a different chemical neighborhood), resulting in about 89 chemically unique $\mathrm{Ag}$ sites. The adsorbate binding energy at an Ag site (and hence the catalytic activity of that site) not only depends on the chemical identities of its neighbors, but also the distance to these neighbors. Figure 7 shows the distribution of mean distances to the neighbors of Ag sites belonging to the two most prominent clusters on the (111) surface. Clearly, each chemically unique site presents a multitude of physically different sites. As a result, the number of catalytically unique Ag sites far exceeds (>> 89 sites in our simulations) the number of chemically unique Ag sites. Then, the enrichment in one or more elements at the surface (and depletion of other elements) and the concomitant increase in the number of catalytically active sites of that element(s) imply that (a) the number of binding energy peaks are lesser than that for a random distribution of atoms at the surface and (b) the peak intensity and width from the majority element(s) is likely to be much larger than those from the minority element(s).
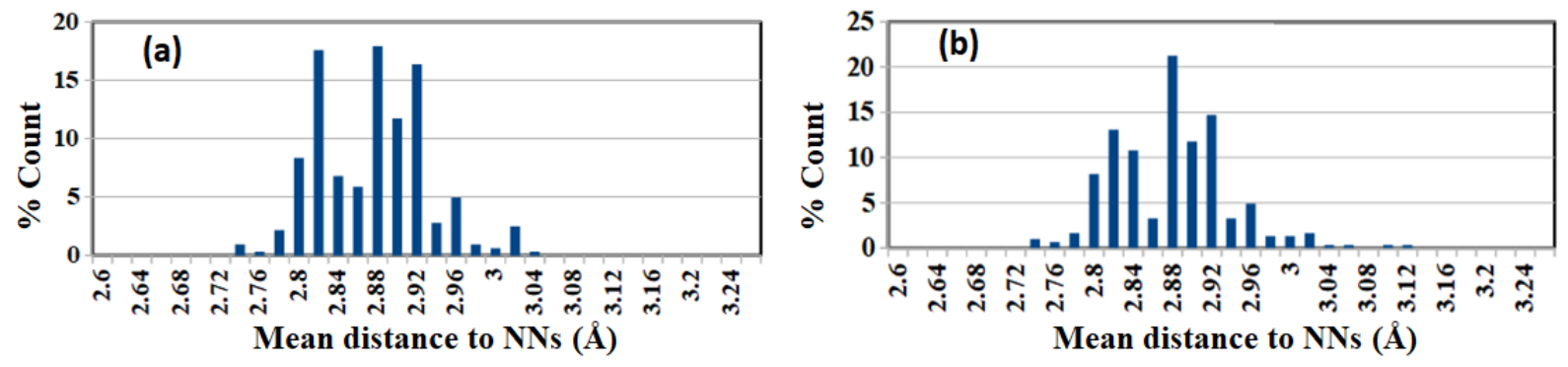

Figure 7: Distribution of mean distances to the neighbors among the two most prominent chemical neighborhoods around an $\mathrm{Ag}$ site on the (111) surface. (a) $(5 \mathrm{Ag}, 1 \mathrm{Au})$ - (2Au, 1Pd) cluster and (b) (5Ag, $1 \mathrm{Au})-(1 \mathrm{Ag}, 1 \mathrm{Au}, 1 \mathrm{Pd})$ cluster.

Overall, results from this study suggest that HEA surfaces may be prone to segregation and that such effects must be an integral part of optimizing the composition of the HEA catalysts to achieve desired activity. Such an effort is currently ongoing in our group wherein machine learning models that account for physical and chemical heterogeneity surrounding a surface site in HEAs are being built to predict adsorbate binding energies for diverse chemical conversions. Using these models to predict the activity of 'segregated surfaces' could then help us perform a more realistic screening of HEA compositions for target reactions. We would like to point out that while this work was in progress, another report appeared in the literature [42] that used abinito calculations to model surface segregation in the AgAuCuPdPt HEA. Results from both the works are consistent and point to the enrichment of $\mathrm{Ag}$ and $\mathrm{Au}$ at the surface of the HEA.

\section{Conclusion}

Embedded atom potential based Monte-Carlo/Molecular-Dynamics simulations were used to study surface segregation in the AgAuCuPdPt high entropy alloy that was recently shown to be active for $\mathrm{CO}_{2}$ reduction. Simulations were carried out on four different surface orientations, (100), (110), (111) and (211), and two different (cubical and spherical) nanoparticles. Analysis of the depth-wise compositional profiles revealed that in all systems, the surface was enriched in $\mathrm{Ag}$ atoms. The concentration of $\mathrm{Ag}$ 
dropped significantly in the subsurface regions. Au was the $2^{\text {nd }}$ most abundant element near the surface, with its concentration peaking in the sub-surface layer. No Pt atoms were found on the surface of either of the six systems. Instead, we observed an enrichment of Pt in the bulk regions. Such segregation pattern seemed to originate from the lower surface energy of $\mathrm{Ag}$ and $\mathrm{Au}$. The compositional mainly gradation occurred over a $\sim 1 \mathrm{~nm}$ region below the surface of the alloy. Beneath this 'near surface' region, the bulk of the material was an alloy of a fixed composition that was enriched in $\mathrm{Pt} \& \mathrm{Cu}$ and depleted in $\mathrm{Ag} \&$ $\mathrm{Au}$. The composition of Pd remained $20 \%$ in the bulk region. Analysis of the neighborhood of each element at the surface revealed that the atoms formed a finite number of chemically unique clusters. The percentage of chemically unique sites was larger for elements with lower concentration at the surface. Together with the physical diversity surrounding each site, the enrichment of $\mathrm{Ag}$ at the surface also increased its number of unique catalytically active sites. Thus, while surface segregation resulted in lower number of distinct elements at the surface, it increased the number of unique catalytically active site for the dominant element. Results from this study strongly suggest that surface segregation and the resultant alterations to the surface atomic structure must be considered an integral part of optimizing the composition of HEA catalysts for any chemical conversion.

\section{Conflict of interest}

There are no conflicts of interest to declare.

\section{Acknowledgement}

This research was supported by the TCS-CTO organization under the SWON number 1009292. We thank the TCS-RnI Infra team for providing necessary support for the execution of this work

\section{Authors' contribution}

C.D., S.G.S and S.M. jointly conceived the work and formulated the implementation plan. C.D. carried out the simulations and their analysis. C.D., S.G.S and S.M. jointly wrote the manuscript. All authors jointly discussed the results and their implications.

\section{References}

[1] Yeh, J.W., Chen, S.K., Lin, S.J., Gan, J.Y., Chin, T.S., Shun, T.T., Tsau, C.H. and Chang, S.Y., 2004. Nanostructured high-entropy alloys with multiple principal elements: novel alloy design concepts and outcomes. Advanced engineering materials, 6(5), pp.299-303.

[2] Cantor, B., Chang, I.T.H., Knight, P. and Vincent, A.J.B., 2004. Microstructural development in equiatomic multicomponent alloys. Materials Science and Engineering: A, 375, pp.213-218.

[3] George, E.P., Raabe, D. and Ritchie, R.O., 2019. High-entropy alloys. Nature reviews materials, 4(8), pp.515-534.

[4] Li, Z., Pradeep, K.G., Deng, Y., Raabe, D. and Tasan, C.C., 2016. Metastable high-entropy dual-phase alloys overcome the strength-ductility trade-off. Nature, 534(7606), pp.227-230.

[5] George, E.P., Curtin, W.A. and Tasan, C.C., 2020. High entropy alloys: A focused review of mechanical properties and deformation mechanisms. Acta Materialia, 188, pp.435-474.

[6] Chen, M.R., Lin, S.J., Yeh, J.W., Chen, S.K., Huang, Y.S. and Tu, C.P., 2006. Microstructure and properties of Al0. 5CoCrCuFeNiTix ( $\mathrm{x}=0-2.0$ ) high-entropy alloys. Materials transactions, 47(5), pp.1395-1401. 
[7] Gludovatz, B., Hohenwarter, A., Catoor, D., Chang, E.H., George, E.P. and Ritchie, R.O., 2014. A fracture-resistant high-entropy alloy for cryogenic applications. Science, 345(6201), pp.1153-1158.

[8] Ding, J., Yu, Q., Asta, M. and Ritchie, R.O., 2018. Tunable stacking fault energies by tailoring local chemical order in CrCoNi medium-entropy alloys. Proceedings of the National Academy of Sciences, 115(36), pp.8919-8924.

[9] Chen, S., Aitken, Z.H., Pattamatta, S., Wu, Z., Yu, Z.G., Srolovitz, D.J., Liaw, P.K. and Zhang, Y.W., 2021. Simultaneously enhancing the ultimate strength and ductility of high-entropy alloys via short-range ordering. Nature communications, 12(1), pp.1-11.

[10] Xia, S.Q., Yang, X., Yang, T.F., Liu, S. and Zhang, Y., 2015. Irradiation resistance in Al x CoCrFeNi high entropy alloys. Jom, 67(10), pp.2340-2344.

[11] Kumar, N.K., Li, C., Leonard, K.J., Bei, H. and Zinkle, S.J., 2016. Microstructural stability and mechanical behavior of FeNiMnCr high entropy alloy under ion irradiation. Acta Materialia, 113, pp.230244.

[12] Luo, H., Li, Z. and Raabe, D., 2017. Hydrogen enhances strength and ductility of an equiatomic high-entropy alloy. Scientific reports, 7(1), pp.1-7.

[13] Qiu, Y., Gibson, M.A., Fraser, H.L. and Birbilis, N., 2015. Corrosion characteristics of high entropy alloys. Materials science and technology, 31(10), pp.1235-1243.

[14] Shi, Y., Yang, B. and Liaw, P.K., 2017. Corrosion-resistant high-entropy alloys: A review. Metals, 7(2), p.43.

[15] Koželj, P., Vrtnik, S., Jelen, A., Jazbec, S., Jagličić, Z., Maiti, S., Feuerbacher, M., Steurer, W. and Dolinšek, J., 2014. Discovery of a superconducting high-entropy alloy. Physical review letters, 113(10), p.107001.

[16] Batchelor, T.A., Pedersen, J.K., Winther, S.H., Castelli, I.E., Jacobsen, K.W. and Rossmeisl, J., 2019. High-entropy alloys as a discovery platform for electrocatalysis. Joule, 3(3), pp.834-845.

[17] Löffler, T., Savan, A., Garzón-Manjón, A., Meischein, M., Scheu, C., Ludwig, A. and Schuhmann, W., 2019. Toward a paradigm shift in electrocatalysis using complex solid solution nanoparticles. ACS Energy Letters, 4(5), pp.1206-1214.

[18] Pedersen, J.K., Batchelor, T.A., Yan, D., Skjegstad, L.E.J. and Rossmeisl, J., 2021. Surface electrocatalysis on high-entropy alloys. Current Opinion in Electrochemistry, 26, p.100651.

[19] Löffler, T., Ludwig, A., Rossmeisl, J. and Schuhmann, W., 2021. What Makes High-Entropy Alloys Exceptional Electrocatalysts?. Angewandte Chemie International Edition, 60(52), pp.26894-26903.

[20] Li, Y. J., A. Savan, A. Kostka, H. S. Stein, and A. Ludwig. "Accelerated atomic-scale exploration of phase evolution in compositionally complex materials." Materials Horizons 5, no. 1 (2018): 86-92.

[21] Li, L., Kamachali, R.D., Li, Z. and Zhang, Z., 2020. Grain boundary energy effect on grain boundary segregation in an equiatomic high-entropy alloy. Physical Review Materials, 4(5), p.053603.

[22] Wynblatt, P. and Chatain, D., 2019. Modeling grain boundary and surface segregation in multicomponent high-entropy alloys. Physical Review Materials, 3(5), p.054004. 
[23] Ferrari, A. and Körmann, F., 2020. Surface segregation in Cr-Mn-Fe-Co-Ni high entropy alloys. Applied Surface Science, 533, p.147471.

[24] Chatain, D. and Wynblatt, P., 2021. Surface segregation in multicomponent high entropy alloys: Atomistic simulations versus a multilayer analytical model. Computational Materials Science, 187, p.110101.

[25] Ledieu, J., Feuerbacher, M., Thomas, C., de Weerd, M.C., Šturm, S., Podlogar, M., Ghanbaja, J., Migot, S., Sicot, M. and Fournée, V., 2021. The (110) and (320) surfaces of a Cantor alloy. Acta Materialia, 209, p.116790.

[26] Nellaiappan, S., Katiyar, N.K., Kumar, R., Parui, A., Malviya, K.D., Pradeep, K.G., Singh, A.K., Sharma, S., Tiwary, C.S. and Biswas, K., 2020. High-entropy alloys as catalysts for the CO2 and CO reduction reactions: Experimental realization. ACS Catalysis, 10(6), pp.3658-3663.

[27] Pedersen, J.K., Batchelor, T.A., Bagger, A. and Rossmeisl, J., 2020. High-entropy alloys as catalysts for the $\mathrm{CO} 2$ and $\mathrm{CO}$ reduction reactions. Acs Catalysis, 10(3), pp.2169-2176.

[28] Zhou, X.W., Johnson, R.A. and Wadley, H.N.G., 2004. Misfit-energy-increasing dislocations in vapor-deposited CoFe/NiFe multilayers. Physical Review B, 69(14), p.144113.

[29] Mishra, S., Maiti, S., Dwadasi, B.S. and Rai, B., 2019. Realistic microstructure evolution of complex Ta-Nb-Hf-Zr high-entropy alloys by simulation techniques. Scientific reports, 9(1), pp.1-11.

[30] Plimpton, S., 1995. Fast parallel algorithms for short-range molecular dynamics. Journal of computational physics, 117(1), pp.1-19.

[31] Humphrey, W., Dalke, A. and Schulten, K., 1996. VMD: visual molecular dynamics. Journal of molecular graphics, 14(1), pp.33-38.

[32] Wouda, P.T., Schmid, M., Nieuwenhuys, B.E. and Varga, P., 1998. STM study of the (111) and (100) surfaces of PdAg. Surface science, 417(2-3), pp.292-300.

[33] Zhao, M., Sloof, W.G. and Böttger, A.J., 2018. Modelling of surface segregation for palladium alloys in vacuum and gas environments. International Journal of Hydrogen Energy, 43(4), pp.2212-2223.

[34] Dowben, P.A., Miller, A.H. and Vook, R.W., 1987. Surface segregation from gold alloys. Gold Bulletin, 20(3), pp.54-65.

[35] Zhao, M., Brouwer, J.C., Sloof, W.G. and Böttger, A.J., 2020. Surface segregation of ternary alloys: effect of the interaction between solute elements. Advanced Materials Interfaces, 7(6), p.1901784.

[36] Patra, A., Bates, J.E., Sun, J. and Perdew, J.P., 2017. Properties of real metallic surfaces: Effects of density functional semilocality and van der Waals nonlocality. Proceedings of the National Academy of Sciences, 114(44), pp.E9188-E9196.

[37] Wynblatt, P. and Ku, R.C., 1977. Surface energy and solute strain energy effects in surface segregation. Surface Science, 65(2), pp.511-531.

[38] Wynblatt, P. and Landa, A., 1999. Computer simulation of surface segregation in ternary alloys. Computational materials science, 15(2), pp.250-263.

[39] Miedema, A.R., 1973. Simple model for alloys. Philips Tech. Rev, 33(6), pp.149-160. 
[40] Miedema, A.R., 1973. Simple Model for Alloys. Pt. 2. Influence of Ionicity on the Stability and Other Physical Properties of Alloys. Philips Tech. Rev., 33(7), pp.196-202.

[41] Dębski, A., Dębski, R. and Gąsior, W., 2014. New features of Entall database: comparison of experimental and model formation enthalpies. Archives of Metallurgy and Materials, 59.

[42] Kristoffersen, H. and Rossmeisl, J., 2022. Local order in AgAuCuPdPt high entropy alloy surfaces. DOI: $10.26434 /$ chemrxiv-2022-b3jqz 